\title{
An evaluation of the CUSUM and inverse velocity methods of failure prediction based on two open pit instabilities in the Pilbara
}

\author{
J. Venter Rio Tinto Iron Ore, Australia
}

A. Kuzmanovic Rio Tinto Iron Ore, Australia

S.D.N. Wessels Rio Tinto Iron Ore, Australia

\begin{abstract}
Predicting the expected time of slope collapse is an important aspect of managing open pit slope stability as it determines the appropriate actions to be taken. It is important to know when to evacuate but is also useful to know well in advance if a particular slope is creeping towards collapse or whether the deformations measured are unlikely to result in collapse. Having this type of information well in advance allows a mine to plan and execute remedial actions, such as schedule changes, slope angle changes and buttresses, that will mitigate economic as well as safety risks. While several methods of analysing slope monitoring data have been published to date, none have been able to establish themselves as the definite answer to any of these questions.

This paper evaluates several previously published methods of predicting the time of slope collapse based on the monitoring data collected for slope instabilities that occurred at two of Rio Tinto's Pilbara Iron Ore operations in 2009 and 2010. The methods tested against the data are: CUSUM, inverse velocity and the slope of velocity and time multiplied velocity (SLO) method (Mufundirwa and Fujii, 2008). The paper concludes by evaluating the effectiveness of each of these methods to serve as early warning of impending failure and to predict the onset of collapse.
\end{abstract}

Both instabilities were managed without injury to personnel and no loss of equipment.

\section{Introduction}

Predicting the expected time of slope collapse is an important aspect of managing open pit slope stability as it determines the appropriate actions to be taken. It is important to know when to evacuate but is also useful to know well in advance if a particular slope is creeping towards collapse or whether the deformations measured are unlikely to result in collapse. Having this type of information well in advance allows a mine to plan and execute remedial actions, such as unloading of slopes or building of buttresses, that will mitigate economic as well as safety risks. While several methods of analysing slope monitoring data have been published to date, none have been able to establish themselves as the definite answer to any of these questions.

This paper evaluates several previously published methods of analysing slope deformation data against two slope instabilities that occurred in the Pilbara. The two slope instabilities occurred at Tom Price's North Deposit (NTD) in 2009 and West Angelas' Centre Pit North (CEPN) in 2010. Both of these slope collapses were managed without harm to personnel or equipment.

The basic failure mechanism for both NTD and CEPN was very similar in that failure occurred on a bedding parallel shale band with stability maintained by several metres of banded iron stone (BIF) preventing the shale band from day lighting. In each case the slope instability was triggered by a reduction in the thickness and quality of the BIF buttress. 
The slope data analysis methods evaluated in this paper are:

- CUSUM (Sarunic and Lilly, 2006).

- Inverse velocity (Rose and Hungr, 2007).

- Slope of velocity and time multiplied velocity (SLO) (Mufundirwa and Fujii, 2008).

During analysis of slope deformation data many pertinent questions are generated. Four questions Geotechnical Engineers are often required to answer when evaluating slope monitoring data were used to test the usefulness of the data analysis methods. These were:

- Can a useful long term prediction of a slope collapse date be made?

- Can a useful short term prediction of a slope collapse date be made?

- Can changes in trend be identified that may lead to collapse?

- Can the method be used as early warning to trigger evacuation?

This paper evaluates each of the three slope data analysis methods based on the two case studies.

\section{Slope displacement data analysis methods}

Slope displacement data has historically been analysed by plotting displacement against time. While continuing to be useful, displacement alone has not been able to predict potential collapse dates. It has also been insensitive to trends in some cases. Recently, three new methods have been published: CUSUMS, inverse velocity and SLO.

Before presenting details of the methods, below is a short synopsis of prism monitoring:

Prism monitoring is carried out by placing a prism as a semi-permanent survey target on a slope to act as laser reflector. Prism locations are measured on a regular basis by setting up a Total Station (Survey Instrument) at a survey pillar with known location. The position in three dimensions of each prism is calculated by taking three measurements. The first measurement is the distance from the pillar to the prism and is called the: 'slope distance' in prism terminology. The second and third measurements are: the angle in the horizontal plane between north and the prism, and the angle in the vertical plane between horizontal and the prism. These angles are called the: 'horizontal angle' and 'vertical angle' respectively. Since three readings are available the coordinates of the prism can be calculated using triangulation. In practice however it is customary to use the following calculations for monitoring purposes:

- Slope distance - The slope distance is often preferred as it is the most precise measurement i.e. it shows the least amount of scatter as laser distance measuring is more precise than mechanical angle measurement. It has the disadvantage that only movement toward and away from the survey instrument can be measured.

- 3D displacement - The 3D displacement is calculated using triangulation of all three measurements. It can measure movement along any of the axis and so eliminates the disadvantage of measuring Slope distance alone. As it is reliant on angle measurements, 3D displacement plots are less precise than slope distance plots as they have more noise and so trends can be more difficult to detect.

- Easting, northing and elevation (RL) - Similar to 3D displacement easting northing and elevation is calculated by triangulating the slope distance and angle measurements. As a result, these values suffer from the same limitations as 3D displacement.

The methods discussed in this paper are based on the slope distance as the selected prisms were able to display the desired movement in slope distance terms and the improved precision of slope distance measurements helped to reduce noise.

The following sections provide a short summary of each of these methods. 


\subsection{CUSUM}

The CUSUM method was published by Sarunic and Lilly (2006) as a tool to interpret slope monitoring data. The CUSUM technique involves summing the difference between the time series data and an arbitrary reference velocity for the time series. This method can be applied to either displacement data or velocity data. The argument for a tool such as this one is that: should the incremental differences tend to one side, a trend will establish itself and become apparent. Sarunic and Lilly (2006) claim that using this tool will aid with the early detection of trends and changes in slope behaviour.

\section{$2.2 \quad$ Inverse velocity}

The inverse velocity method was developed by Fukuzono (1985) with follow up articles with specific reference to open pit instability by Rose and Hungr (2007). The basis of the inverse velocity method is to calculate the rate of deformation of the slope (velocity) and to plot the inverse of the rate of deformation against time (inverse velocity against time). As the velocity or rate of deformation increases the inverse will tend towards zero. This provides a clear target for anyone attempting to predict collapse time to extrapolate to as a straight line regression can be used to find the expected horizontal axis intercept. This intercept then presents the expected time of collapse.

\subsection{SLO}

The SLO method was presented by Mufundirwa and Fujii (2010) as a feasible failure time predictor based on work carried out by Fukada, et al. (1999) on the creep behaviour of laboratory tests. The SLO is so named as it uses the slope of the graph obtained by plotting the displacement velocity multiplied by time on the $y$-axis and the displacement velocity on the $x$-axis to predict a failure date. The resulting graph is approximately linear and increases from left to right (Figure 9).

The failure date is obtained by calculating the slope of the resulting graph. This provides an expected date of failure.

In conclusion, three methods for assessing slope monitoring data for two slope failures are presented here, particularly addressing the usefulness of the methods for predicting slope failure and changes in slope movement rates.

\section{$3 \quad$ Methodology}

The prediction tools were evaluated not by hindsight using all available data but by limiting the data to that which was available at the time predictions would have been required. This was done by producing graphs, showing predictions for collapse dates versus prediction dates. These graphs were then compared to actual collapse dates.

The prism data was processed using the following steps:

1. Tabulate the measured slope distance data against date measured.

2. In cases where obvious survey errors were made, these points were filtered out of the dataset.

3. Calculate the displacement by subtracting the first slope distance measurement in the table from each subsequent slope distance value. These values were then plotted as slope distance displacement versus date.

4. The displacement rate or velocity was calculated by taking the slope of the last eight measurements (four weeks) in the case of NTD, and 16 measurements ( 16 days) in the case of CEPN, and carrying out a linear regression analysis as suggested by Rose and Hungr (2007). Rose and Hungr (2007) did not specify how many measurements to use and these values were selected to provide a reasonably smooth velocity curve with few negative values which is a requirement for calculating the inverse velocity method. Rose and Hungr (2007) did envisage that the time over which the velocity should be averaged is a function of survey precision and is influenced by 
random variables such as diurnal effects. They, Rose and Hungr (2007), recommend that the number of readings over which velocity must be averaged should be determined experimentally by selecting a value that provides a smooth curve without hiding trends.

5. The inverse velocity was calculated by inverting the velocity.

6. The cumulative sums for the CUSUM method were calculated based on the velocity information.

7. Finally the SLO graph was generated by multiplying the velocity by the number of days since the first reading to obtain a value for the $y$-axis. The velocity for the $x$-axis was obtained earlier. Using this method the slope of the curve then presents the predicted failure date as a number of days since the first reading in the data table. For this paper these were presented as dates to simplify presentation.

\section{$4 \quad$ Case studies}

The monitoring analysis methods discussed in this paper were tested on data obtained from two open pit slope collapses that occurred in the Pilbara. The first collapse occurred in the North Deposit Pit at the Tom Price Mine Site (NTD) and the second collapse took place in the Centre Pit North at the West Angeles Mine Site (CEPN). Both slope instabilities were managed without harm to personnel or equipment.

This paper limits its discussion of these instabilities, to the selected monitoring data only, to keep the paper manageable and stay on topic.

\subsection{Tom Price - North Deposit (NTD)}

The east slope of the NTD Pit collapsed on 28 September 2009 (see Figure 1). The failure mechanism was structurally controlled by a polished shale band parallel to bedding which dipped into the pit at $26^{\circ}$ and parallel to the overall slope angle. The resulting landslide created approximately 1 million tonnes of failed material. The total slope height was $40 \mathrm{~m}$ at the time of collapse.

The prism data presented in this paper is based on prism monitoring twice a week from the beginning of the dataset followed by daily measurements from 22 September 2009. Only four prisms were selected for presentation in this paper, to avoid cluttering of the graphs. The prisms were selected as those whose slope distance graphs most clearly showed the movement trend of the slope. This was a result of them being near the centre of the instability.

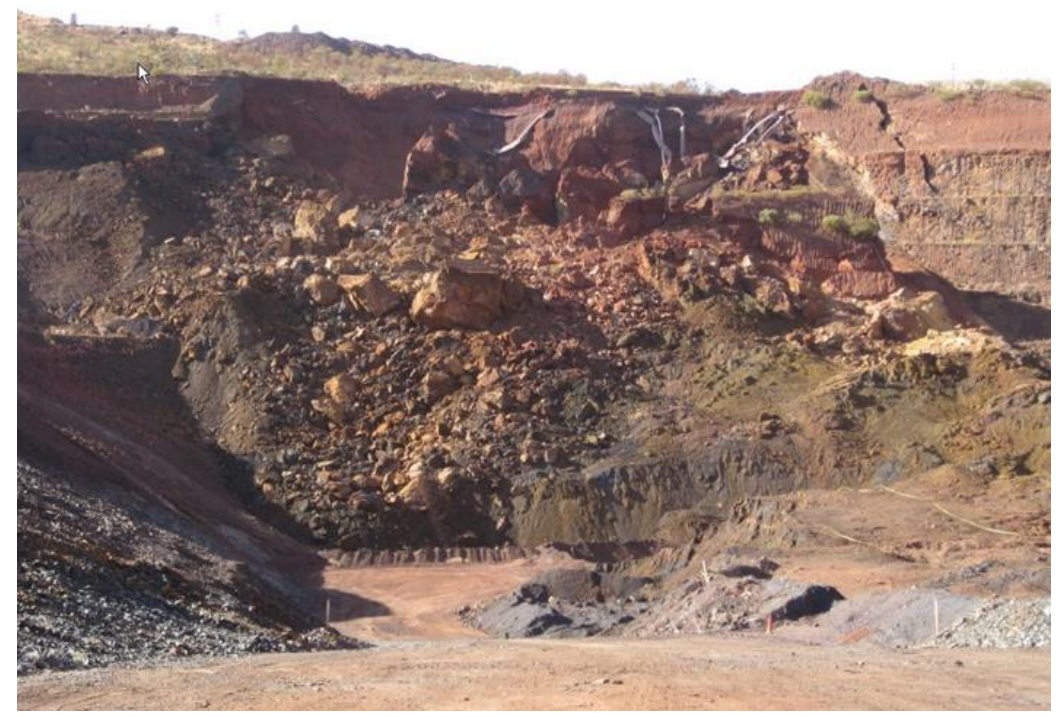

Figure 1 NTD 2009 slope instability 


\subsubsection{Slope distance}

The slope distance for the selected prisms of the NTD 2009 instability is presented as Figure 2.

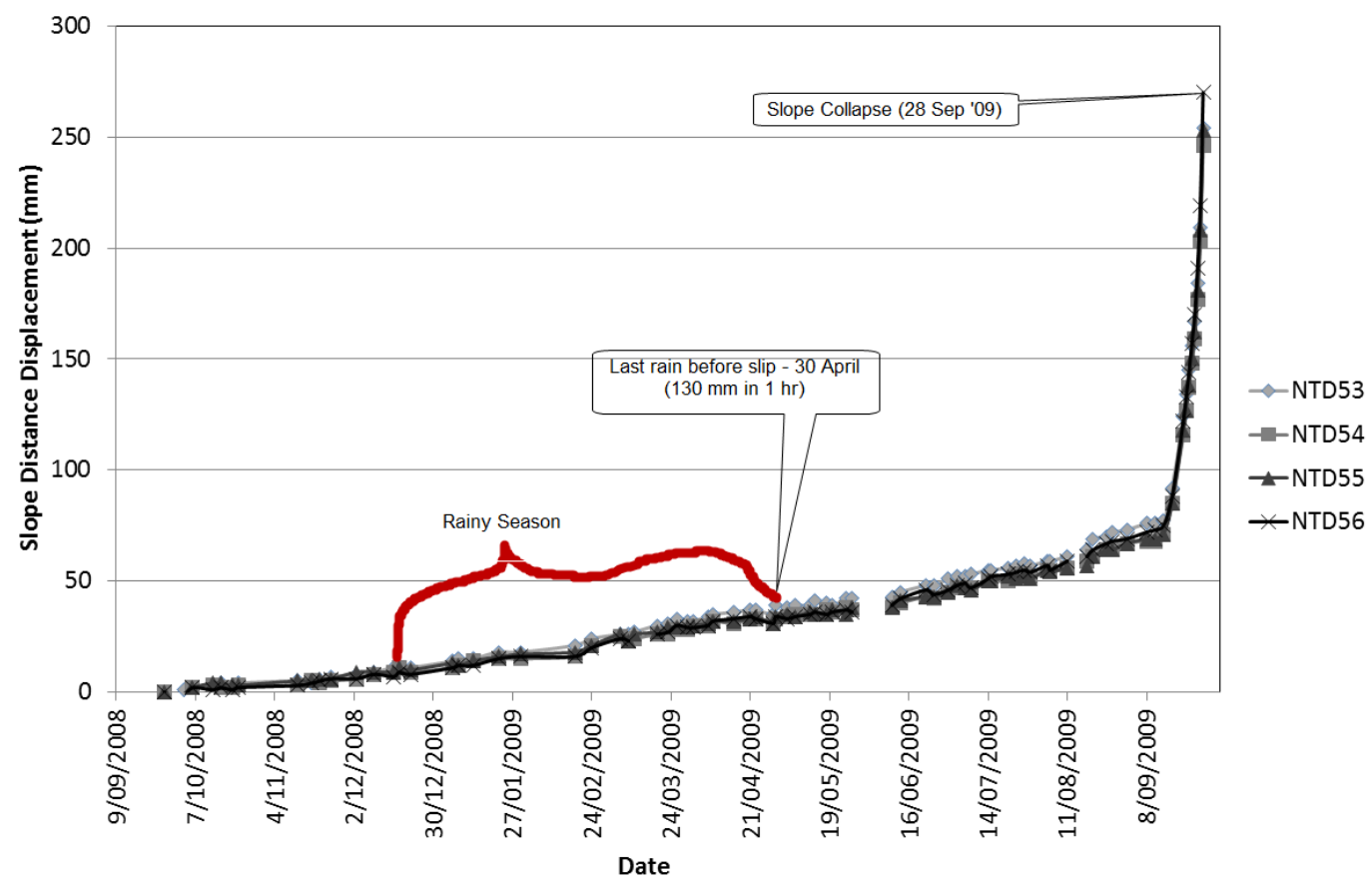

Figure 2 NTD - slope distance versus time

The displacement presented as Figure 2 gives the impression that the slope collapsed without warning. This is however not the case. The large displacement measured in the last days distorts the shape of the graphs due to the exaggerated vertical scale. To correct this error the last few days were clipped and the new graph is presented as Figure 3. It can now be seen that there is a gradual increase in displacement rate which can be correlated to mining closer to the slope. The data in the last few months can be correlated to individual blasting and mining events although these are not presented here.

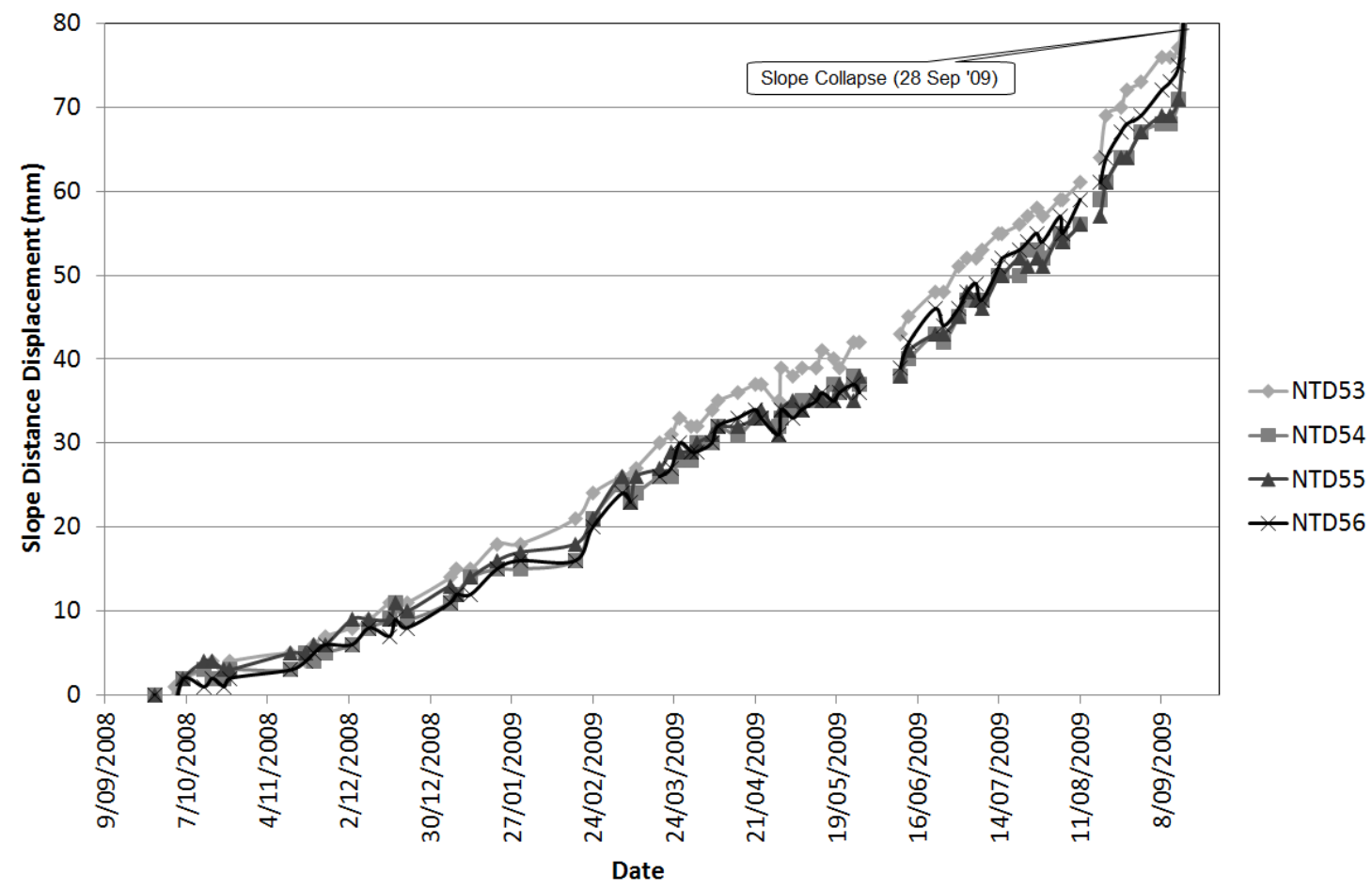

Figure 3 NTD - slope distance with last days removed 
The slope distance data showed several changes in trend over the prism record and was able to provide information indicating acceleration resulting in slope collapse. Interpretation of the slope distance plots are sensitive to graphing scale, consequently slope distance plots must be viewed at multiple scales if correct conclusions are to be drawn.

\subsubsection{Velocity}

The velocity graphs of the individual prisms are presented as Figure 4 . The velocity plot for prisms in NTD is based on linear regression of an 8 day moving window as presented in Section 3. It is clear that there are several periods of acceleration and deceleration over the last year before the slide. Most of these are correlated to mining and non-mining periods although the gradual increase in velocity coincides with the cyclone season and the big drop the end of cyclone season (Figure 4). The best explanation for the gradual increase during 2009 in a period of dry weather is the increase in mining activity in an attempt to deplete the ore in this pit before the next cyclone season.

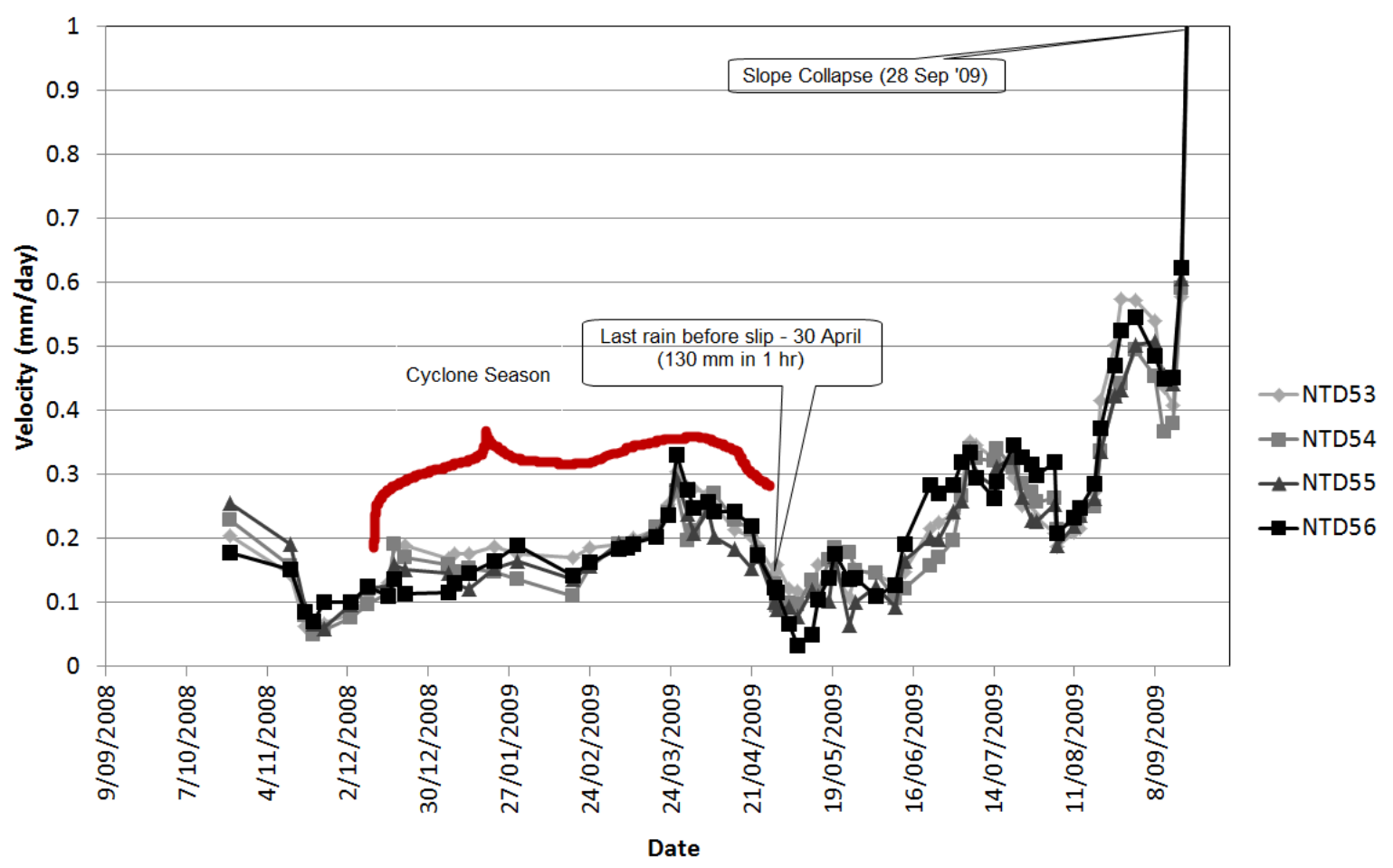

Figure 4 NTD - prism velocity

The velocity information showed a maximum velocity before the onset of slope collapse of $0.55 \mathrm{~mm} /$ day. Such a low value can be explained by the brittle nature of the failure mechanism being driven by sliding on a weak plane. The velocity plot was more useful to identify trends than the slope distance plot and its interpretation was less sensitive to graphing scale errors. It is not clear whether useful alarm or evacuation decisions could be made off the velocity plot as opposed to the slope distance plot.

\subsubsection{CUSUM}

The CUSUM plot for velocity is presented as Figure 5. In the case of NTD, interpretation of the CUSUM plot was not as sensitive to graphing scale as the velocity and slope distance chart and appears to be more sensitive to underlying trends. As a result, the movement trend in the last few months before the slip is better defined in the CUSUM plot as compared to slope distance and velocity plots.

For the Tom Price North Deposit data, interpretation of the CUSUM plot was more sensitive to movement trends than the velocity and slope distance plots and less sensitive to graphing scale distortions. Using linear trend lines it may have been possible to use the CUSUMS for alarming and evacuation decisions. 


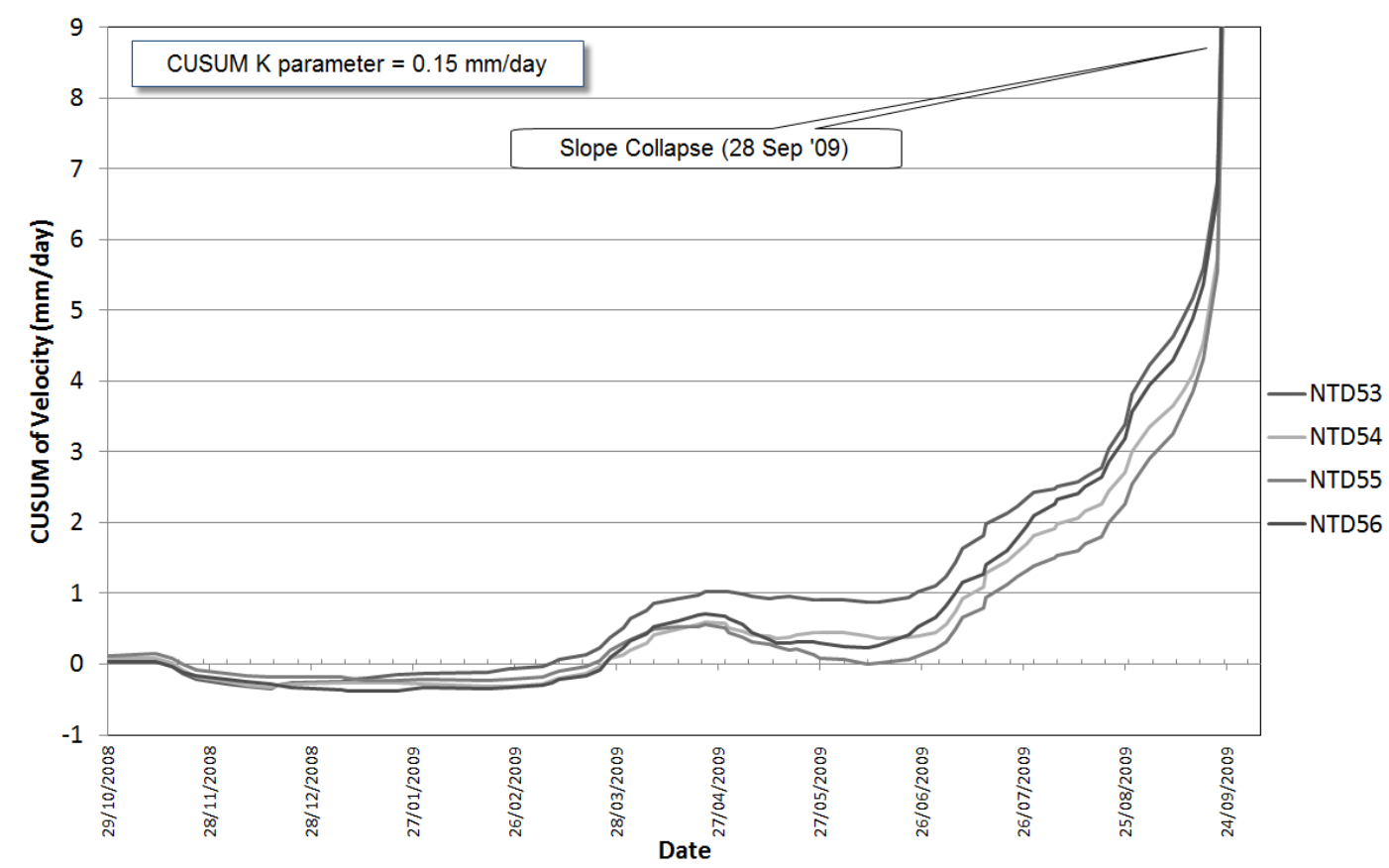

Figure 5 NTD - CUSUM chart

\subsubsection{Inverse velocity}

The inverse velocity plot for a one year history is presented as Figure 6 with the last month of data presented as Figure 7. The Date of Prediction versus Prediction Date plot presented as Figure 8. For the NTD 2009 failure, the inverse velocity plot presents a clean scatter of data that appears to trend well towards the date of slope collapse. Barring a trend change early in 2009 one might well assume that the failure date could be picked by using this method. Unfortunately slopes respond to external drivers such as rain, mining and blasting and any early prediction excludes information about future external events that will influence collapse dates. Figure 8 shows that up to early May 2009 most predictions would be on the 'safe' side in that the failure date would be underestimated. After early May predictions are beyond the actual date of collapse making them unsafe.

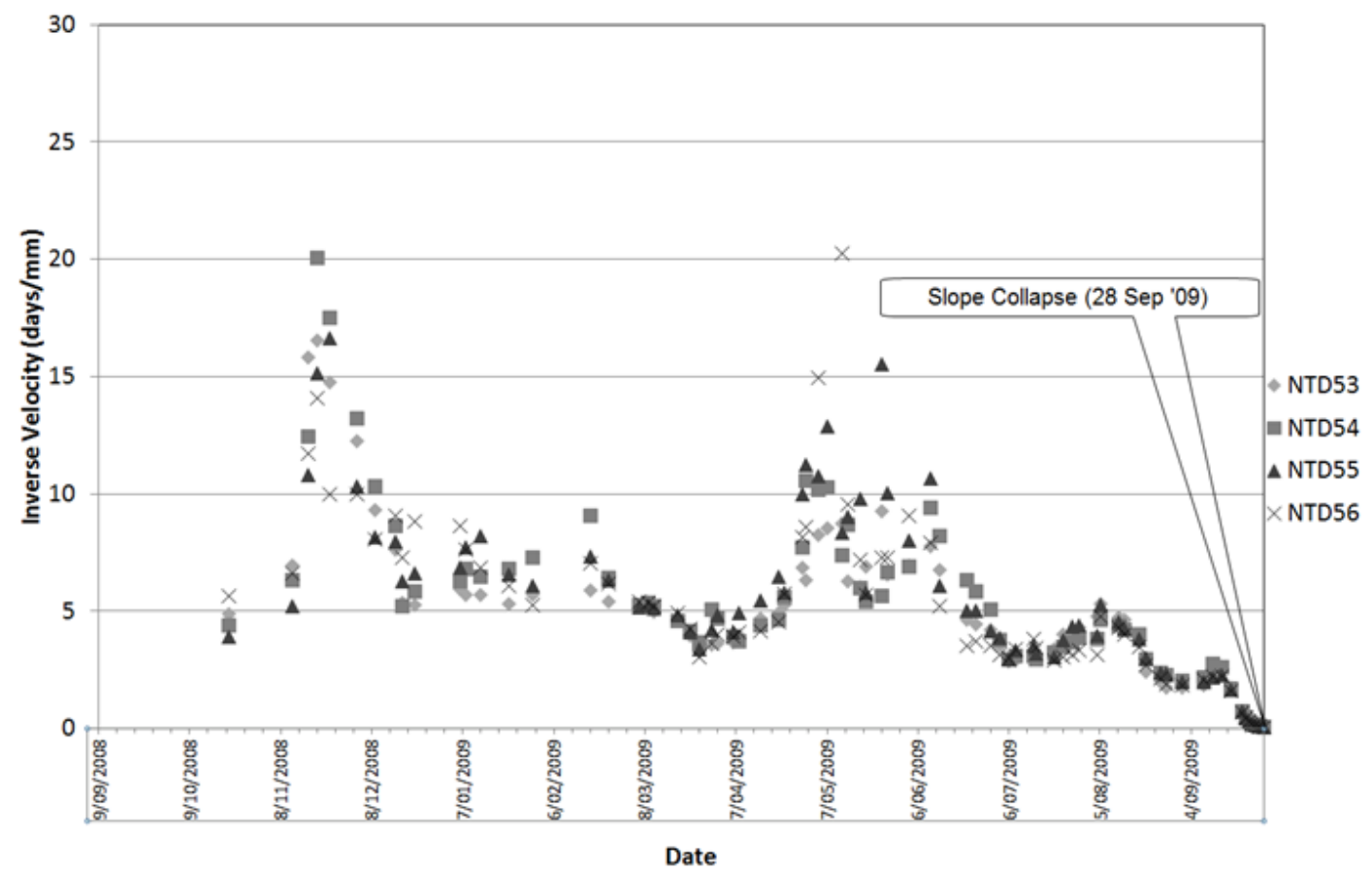

Figure 6 NTD - inverse velocity one year history 
It can easily be accepted that attempting to predict a date of collapse years or even months in advance is unreasonable considering that factors external to the slope has the potential to increase or slow down slope movement. An alternative question is whether the inverse velocity provided a reasonable estimate of collapse date once the slope started accelerating. To investigate this, Figure 7 presents the last month's inverse velocity data prior to collapse. In Figure 7, the last two weeks of data present two different trends. The first one predicting a collapse date on approximately 24 September and the second a collapse date after 28 September, which was the real collapse date. It is noteworthy that the last two weeks' worth of data show a decelerating trend which explains the over prediction of the collapse date in the last week.

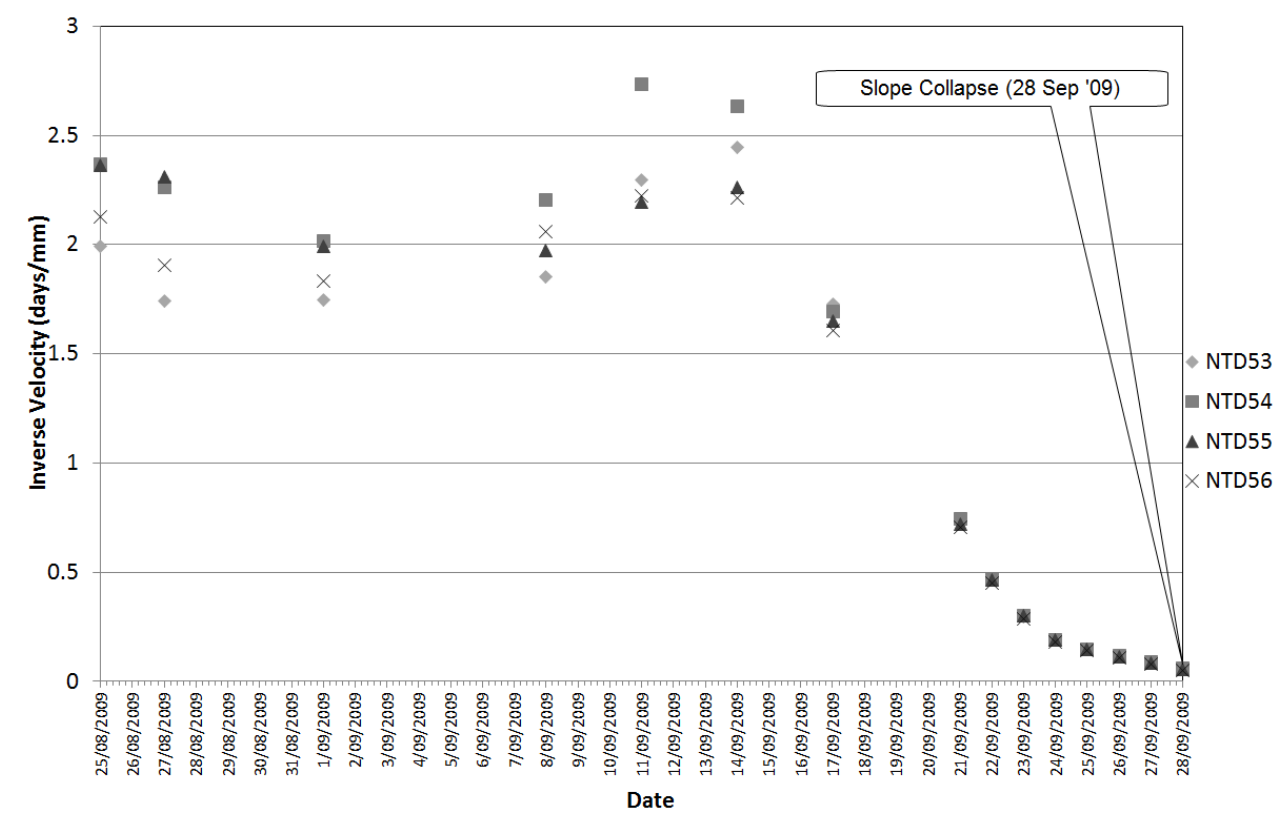

Figure 7 NTD - inverse velocity plot showing last month

The NTD inverse velocity plot is sensitive to scatter in velocity data which required some filtering to remove negative values. The NTD inverse velocity data was sensitive to graphing scale as only the trends predicted shortly before the collapse date has any real meaning and still failed to safely and unambiguously predict a useful collapse date.

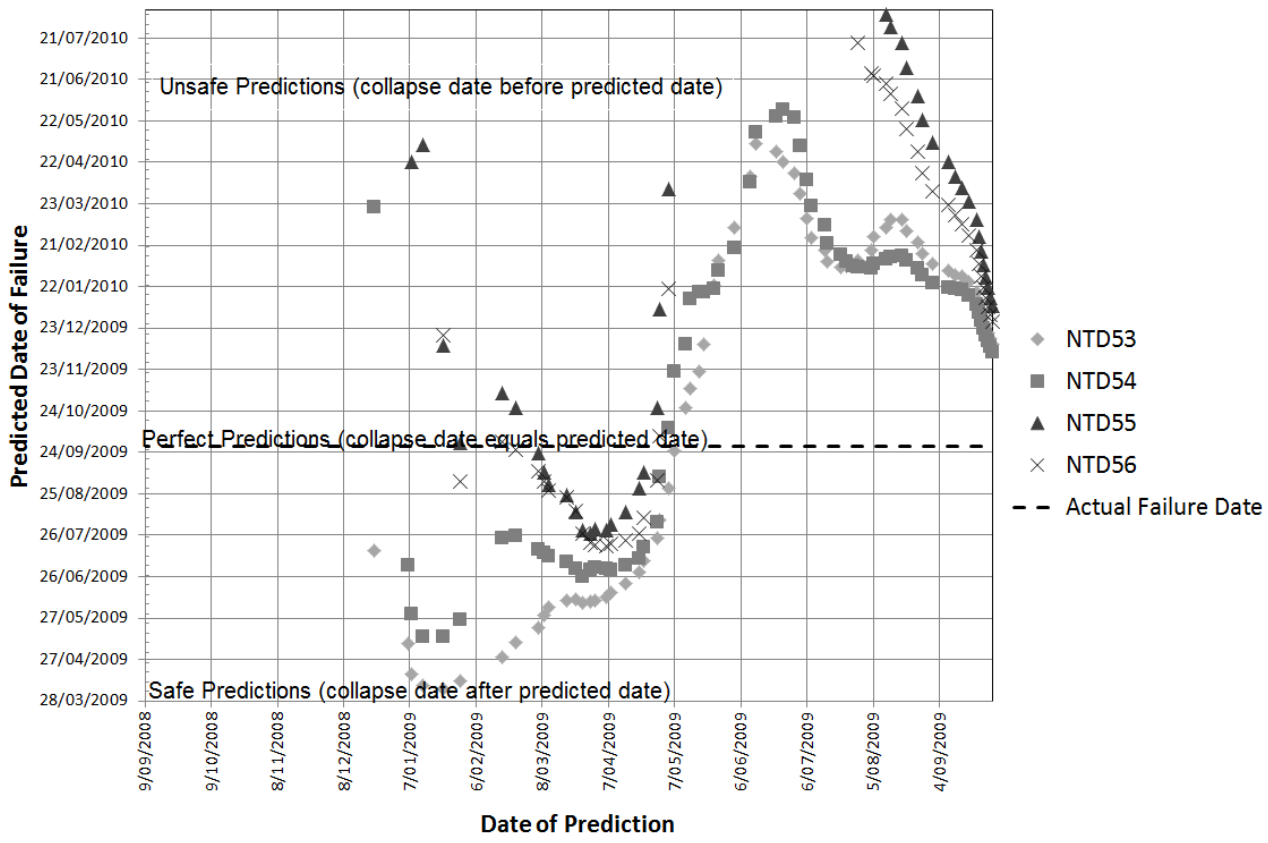

Figure 8 NTD - inverse velocity prediction date versus predicted date of failure 


\subsubsection{SLO}

The results of the SLO analysis are presented as Figure 9 to Figure 11. Figure 9 presents the full history which appears to show a perfect straight line which in theory should mean one predicted date of collapse. Closer inspection revealed that the last few measurements created a false impression of clarity just as it did for the displacement and CUSUM methods.

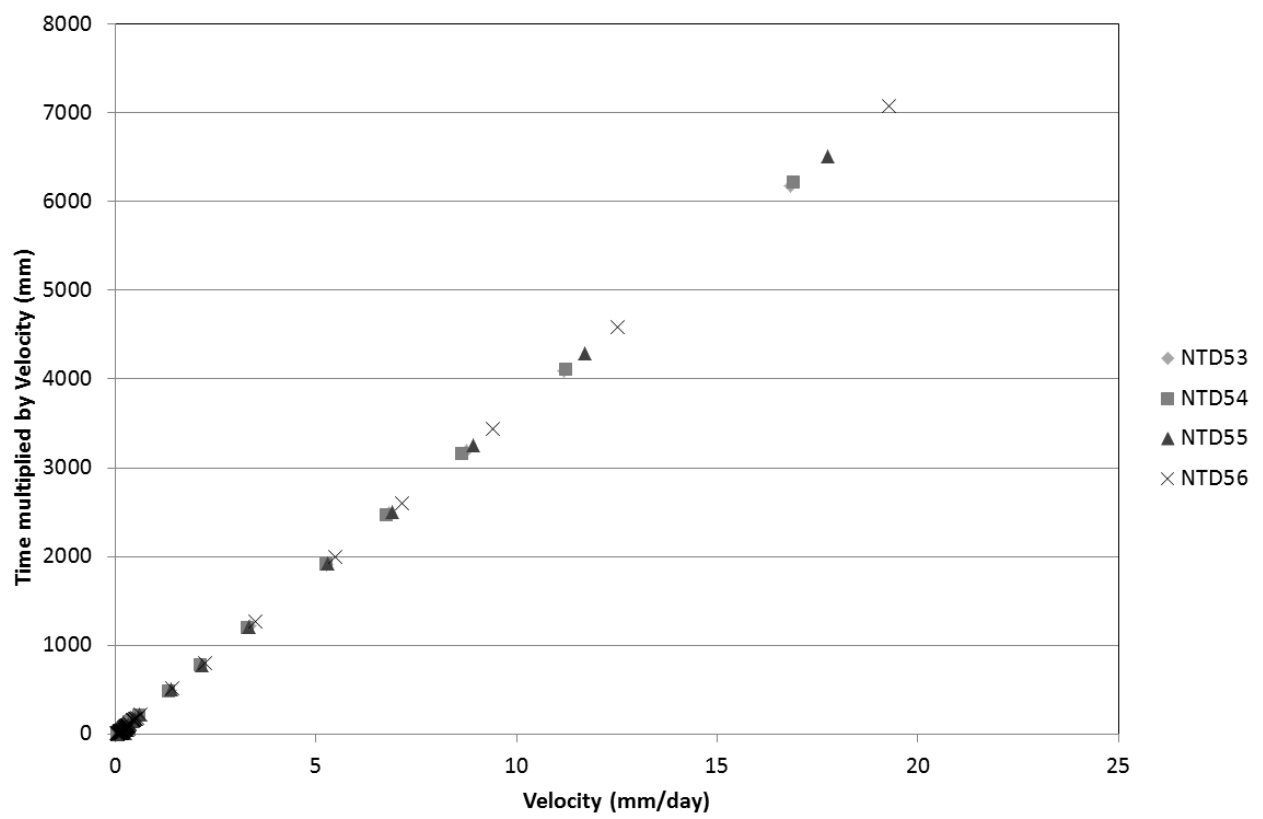

Figure 9 NTD - SLO plot for entire history

Figure 10 presents an enlarged plot with the latest measurements clipped, as such it becomes apparent that at least six different slopes can be observed, each of which would provide a different prediction for a failure date. A similar predicted date versus prediction date analysis as that carried out for the inverse velocity method was carried out through linear regression of all the data points (Figure 11). Figure 11 shows that in the final months before the slip most predictions would have been 'safe' but in the final month the predicted date suddenly falls after the actual slip date making it unsafe. 


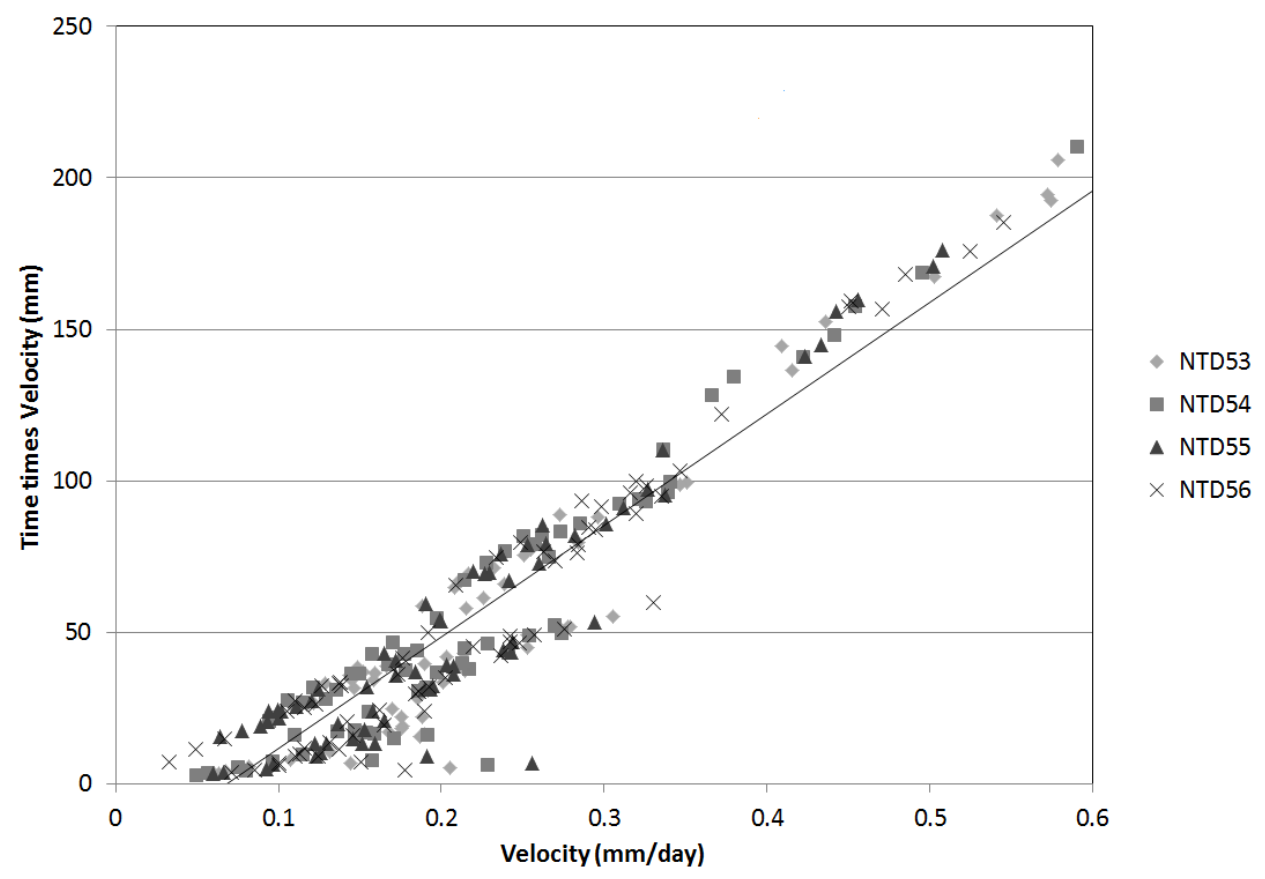

Figure 10 NTD - SLO plot with acceleration to collapse clipped (line shows trend during final days)

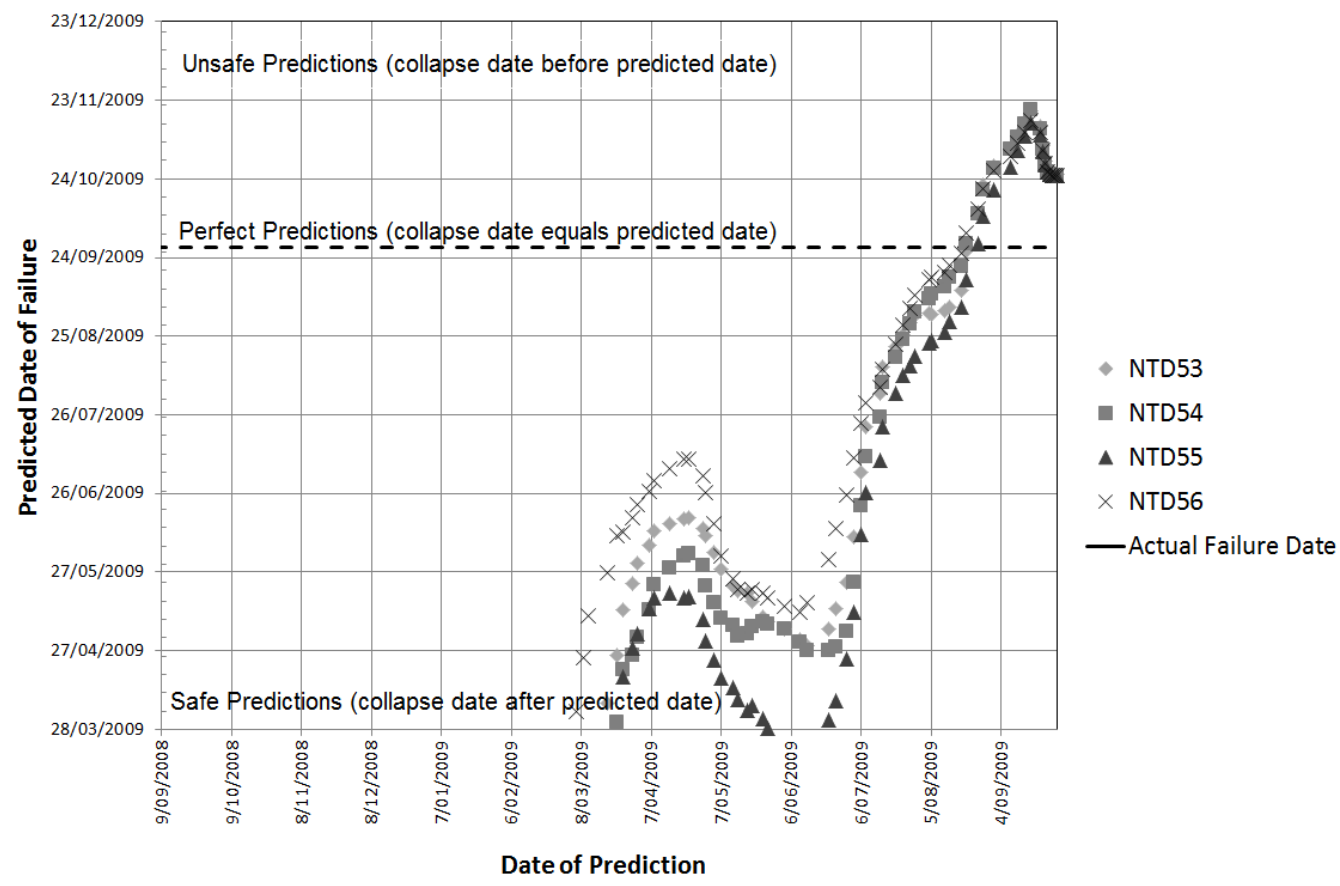

Figure 11 NTD - SLO prediction date versus predicted failure date

Estimation of the collapse date using only the last seven days' worth of data is two days short of the real date on 26 September while using all data prior to collapse is 25 September. Both of these predictions appear to have been useful in that they predicted a collapse date a few days short of the real date. Unfortunately this prediction is based partially on hindsight as the last three days of data were required to establish the trend. The prediction therefore provided a date that had already passed.

In summary, the SLO method proved to be very sensitive to the period over which velocity is calculated. Long periods provided clear data but short periods provided too much scatter in prediction dates. Once an 
accelerating trend was established, the predicted date was two days short of the real date but this estimate was provided with partial hindsight.

\subsection{West Angelas Centre Pit North (CEPN)}

The north slope of CEPN collapsed on 2 February 2010 (see Figure 12 for photo). Similar to NTD, the failure mechanism was sliding on a bedding parallel shale band. At the time of collapse the slope height was $170 \mathrm{~m}$ at an overall angle of $40^{\circ}$.

The prism data presented for CEPN is based on daily averages of an automated survey system providing 6 readings per day. As for NTD, four prisms were selected to present here.

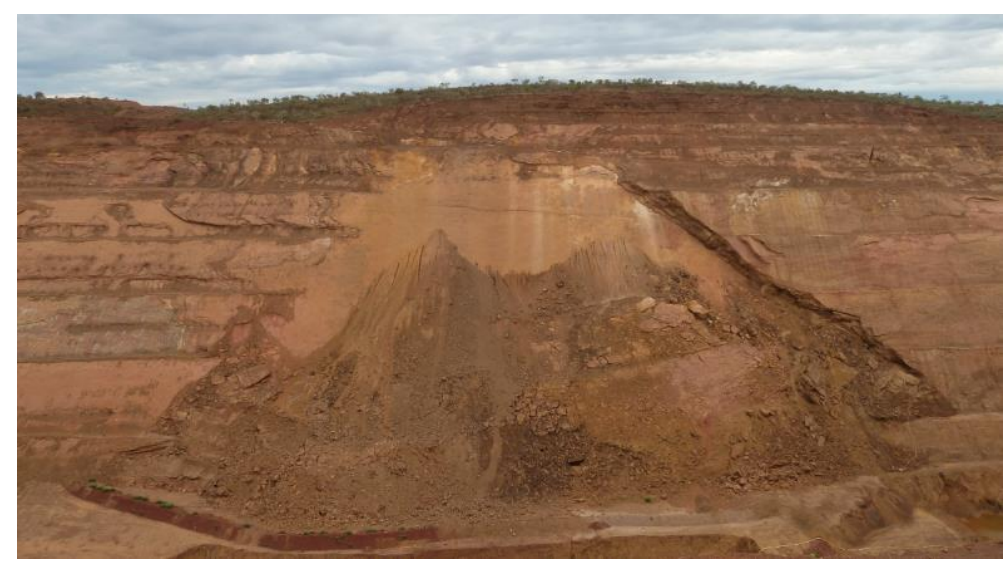

Figure 12 CEPN 2010 slope instability

\subsubsection{Slope distance}

Selected prism monitoring slope distance data for the CEPN slope instability is presented in Figure 13. There appears to be two stick slip events but the final days before the collapse shows a gradual increase in displacement leading up to collapse.

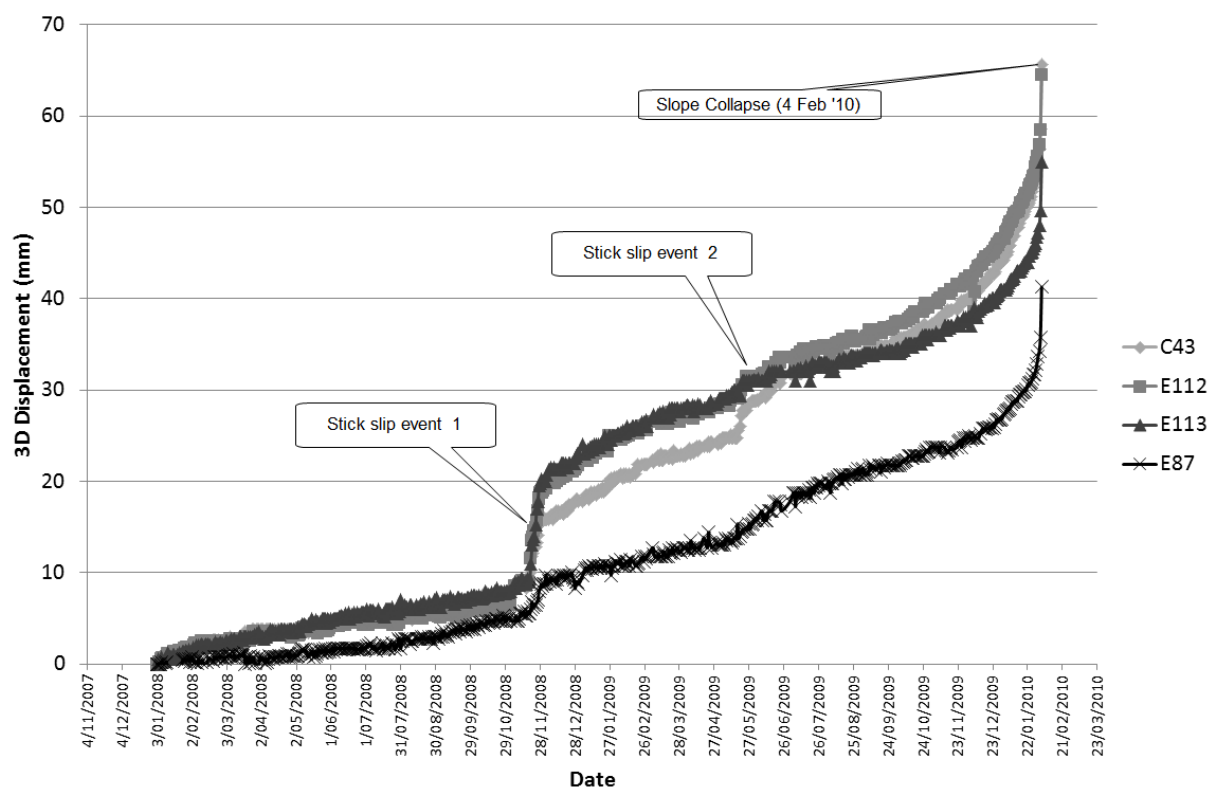

Figure 13 WA - slope distance

Interpretation of the CEPN slope distance plot was sensitive to trend changes but was also sensitive to graphing scale. This made the results difficult to interpret for alarming purposes. 


\subsubsection{Velocity}

The velocity plot for the CEPN 2010 slope failure is presented as Figure 14. There are several acceleration events corresponding to stick slip behaviour but the final months indicate a gradual increase in velocity lasting about two months. It is interesting that the slope showed large velocities in the past during stick slip events of similar magnitude to those near collapse but did not fail. Presumably the BIF buttress preventing shale day lighting was still intact at this stage.

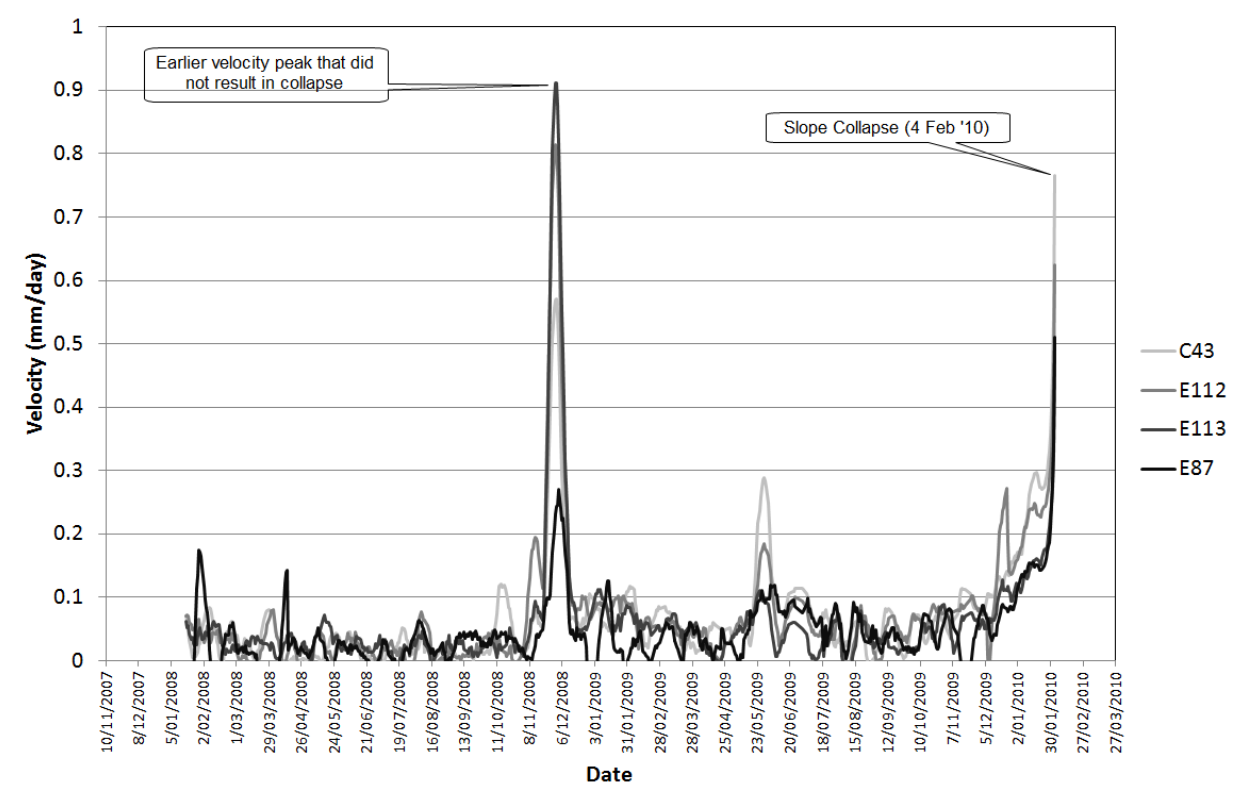

Figure 14 WA - velocity (note the spike in 2008 that did not result in slide)

Interpreting the CEPN velocity plot is sensitive to trend changes but did not provided useful information for evacuation decisions as larger velocities were experienced in the past compared to those at time of collapse. It can be speculated that as mining progressed more of the toe buttress was mined out making the slope more brittle.

\subsubsection{CUSUM}

The CUSUM plot for the CEPN 2010 slope failure presented as Figure 15. In contrast to the velocity plot where the scatter hides underlying trends, four distinct periods can be identified over the history of prism movement. The last of these shows gradual acceleration from early December 2009 to collapse on 4 February 2010. 


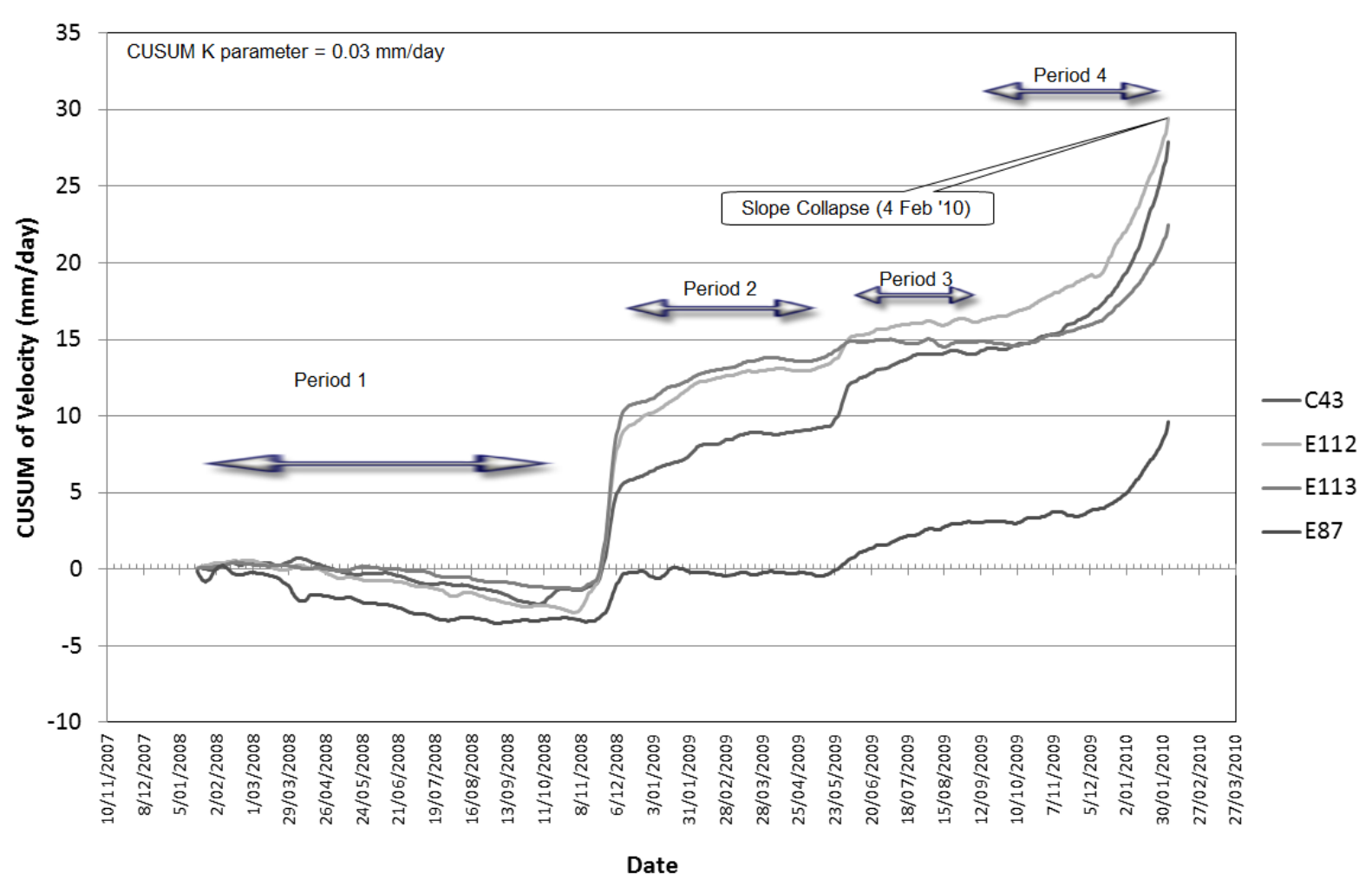

Figure 15 WA 2010 slope failure - cumulative sum (CUSUM) plot

Interpreting the CUSUM plots was not as sensitive to graphing scale distortion as the slope distance plots but was still sensitive to trend changes. Similar to the slope distance velocity plot the gradual acceleration to collapse would have made automated alarming difficult to apply.

\subsubsection{Inverse velocity}

The inverse velocity plot for West Angelas (Figure 16) appears virtually like a random scatter plot in spite of averaging velocities over 16 days. This is a result of the large scatter in survey readings. Two earlier potential failure times can be identified on this plot corresponding to stick slip behaviour.

As a result of the large number of underlying trend changes and the scatter in the survey data, it is doubtful whether a longer term prediction of collapse date could be made using the inverse velocity method. A short term prediction was investigated based on period and data presented in Figure 17. Figure 17 shows the last six weeks of monitoring data prior to slope failure. In this figure, at least four different trends can be determined with prediction dates ranging from never to well short of the actual date. All four prisms showed an accelerating trend in the last two weeks before the collapse. This implies that any prediction made using short term data would have over predicted the collapse date and therefore be unsafe. 


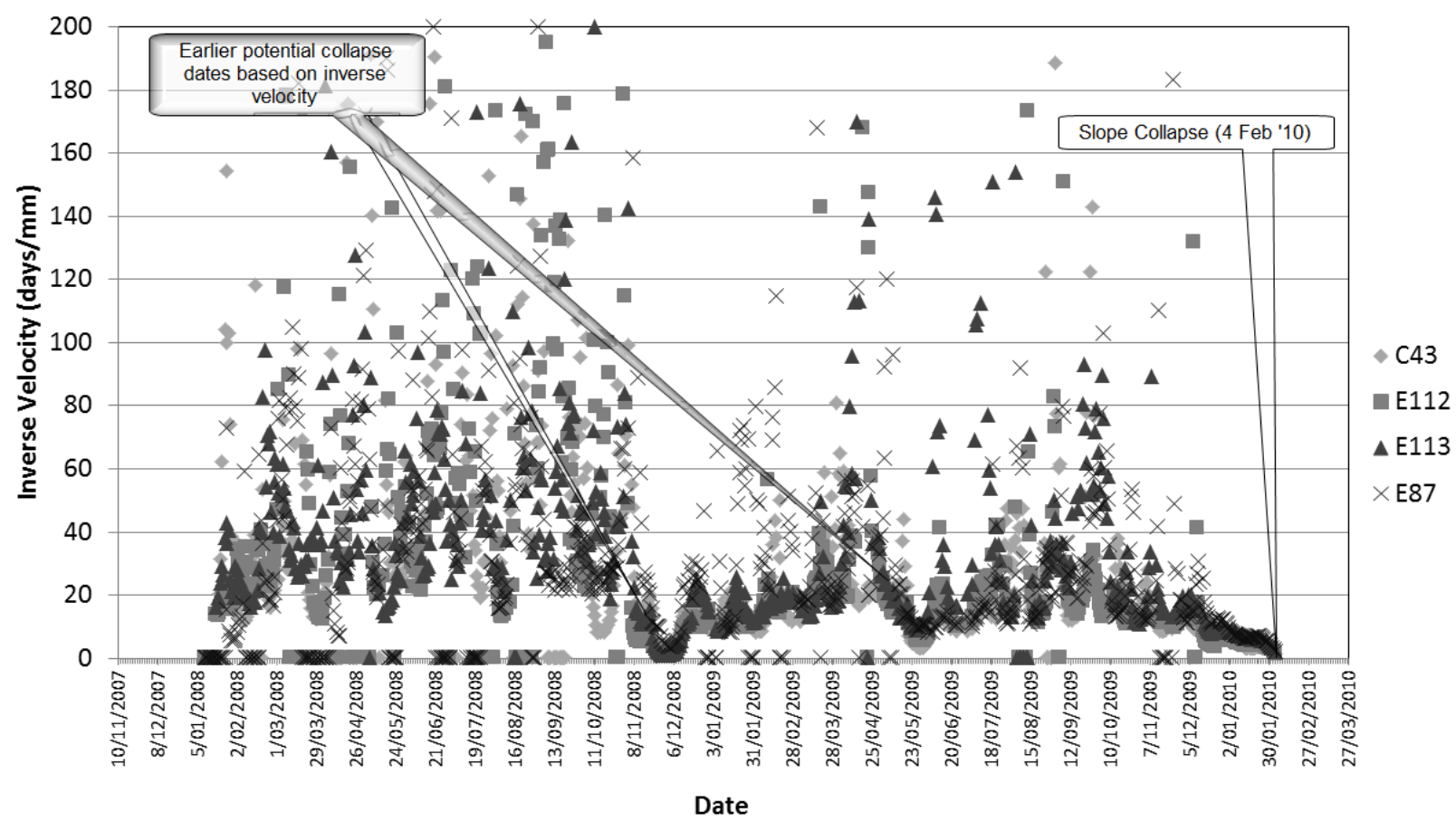

Figure 16 Inverse velocity for entire history for CEPN 2010 slope failure

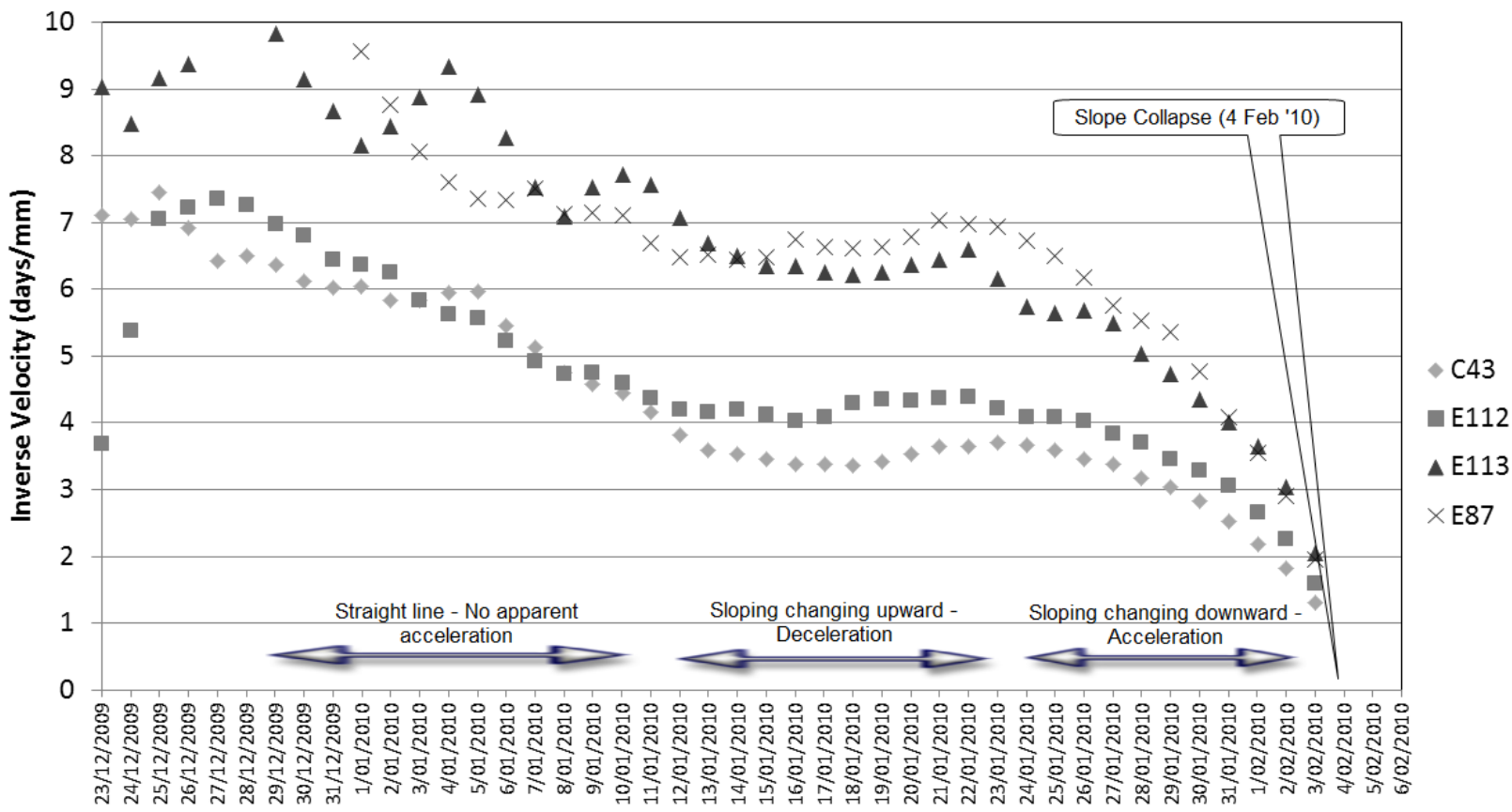

Date

Figure 17 CEPN 2010 slope failure - inverse velocity for the last six weeks

\subsubsection{SLO}

The SLO plot for the selected prisms from the CEPN 2010 slope failure is presented as Figure 18. Five trends can be identified over the monitoring history. One of them is very strong corresponding to the stick slip event in November 2008. The trend that resulted in collapse is marked in the figure. The predicted date using the complete final trend provided an estimated collapse date of 4 February which was two days after the actual date. If only the last seven days' worth of data is used the predicted date is 7 February 2010. 
The SLO method identified the same diversity of trends identified using other methods for the CEPN 2010 slope failure data but due to the scatter failed to provide useful long or short term predictions. In addition, the short term predictions were unsafe in that the collapse date was over predicted.

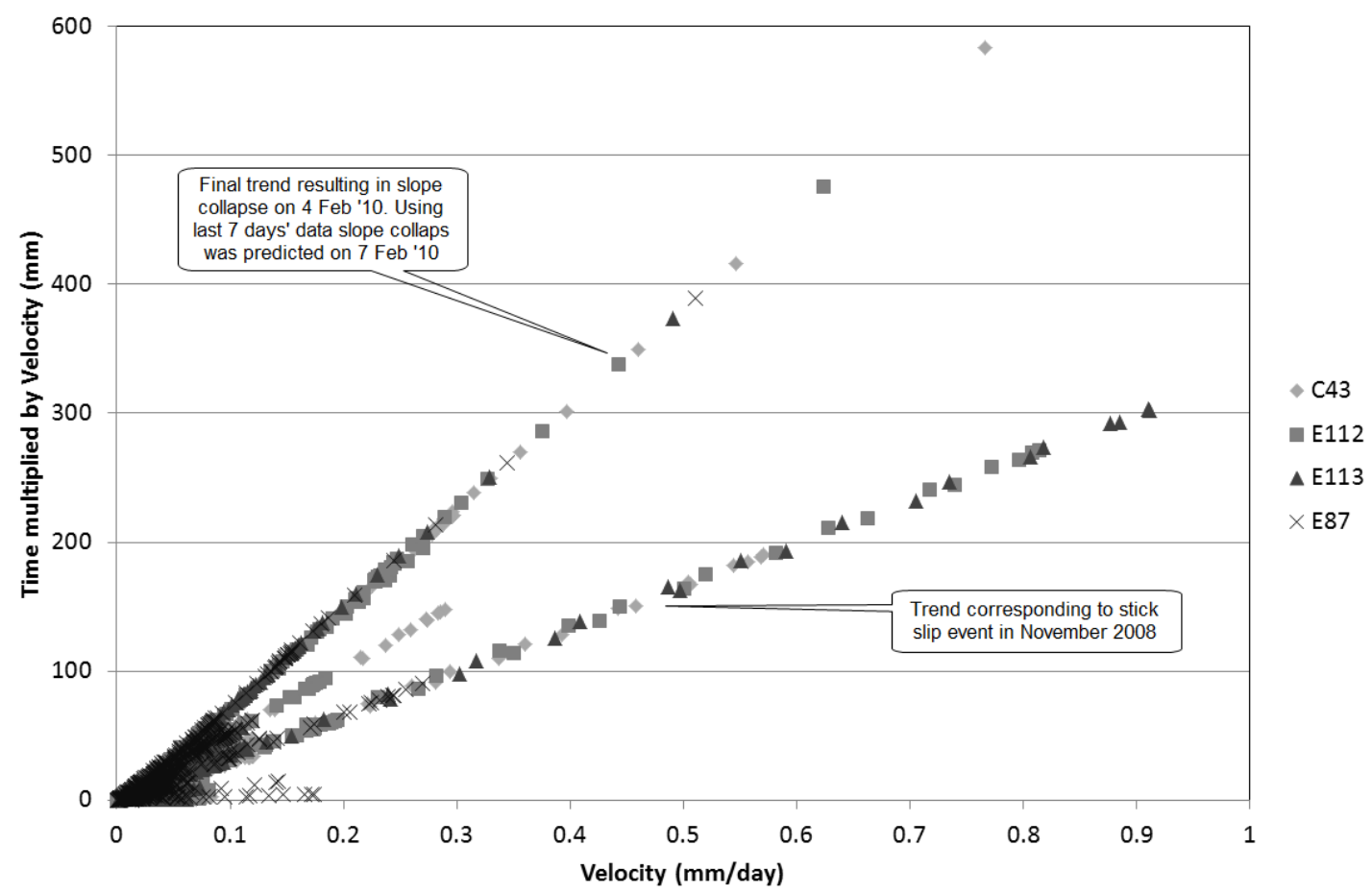

Figure 18 CEPN 2010 slope failure - SLO plot for full History

\section{Discussion}

This paper tested the three slope monitoring data analysis methods (CUSUMS, inverse velocity and SLO) against data sets from two slope instabilities (NTD and CEPN). The performance of these methods was measured against their ability to answer the following four questions:

- Can a useful long term prediction of slope collapse date be made?

- Can a useful short term prediction of slope collapse date be made?

- Can changes in trend be identified that may lead to collapse?

- Can the method be used as early warning to trigger evacuation?

This discussion summarises the performance of these methods against each of the questions.

\section{$5.1 \quad$ CUSUM}

As the CUSUM method makes no claim to being able to predict potential failure dates, in contrast to the inverse velocity method and SLO method, it can safely be concluded that the CUSUM method cannot predict slope collapse dates.

With regard to being able to identify different movement trends, the CUSUM method performed very well as it was able to identify trends in both case studies and may possibly be more sensitive than other methods, even where a high degree of scatter in survey data was present.

The CUSUM method did not provide an absolute value that could be used as alarm for evacuation but could with the aid of trend lines be used to trigger decision points. The last of these decision points would then serve as evacuation trigger. If used in this capacity it must be noted that the need to average data over several measurements in order to calculate velocity for CUSUMS may negate any benefit that a more sensitive method may bring as it will have a lag equal to the number of days the data is averaged over. 


\section{$5.2 \quad$ Inverse velocity}

In the case of NTD the inverse velocity method appeared to present a coherent picture of slope movement but on deeper analysis failed to provide useful predictions of a long term collapse date. The short term collapse date was under predicted. At CEPN where the survey scatter was greater than at NTD the inverse velocity method failed to provide any useful prediction of long term failure date. Using only the last data a close but unsafe prediction of collapse was made.

The inverse velocity information for both case studies allowed some interpretation to be made regarding trends but arguably did not provide more information than what would have been available looking at velocity or displacement. In addition, interpreting the data is very sensitive to selecting the correct averaging period for velocity calculations. It was noticed that if the last few measurements prior to collapse show deceleration, as at NTD, the short term predicted date of collapse is likely to be short of the actual date making it safe (see Figure 17). If the last measurements show acceleration, such as CEPN, the short term predicted data of collapse is likely to be after the actual date, making it unsafe.

Due to the scattered nature of the inverse velocity plots, the need for data averaging to calculate velocity, and the non-linear nature of the vertical axis, it is doubtful that useful evacuation triggers can be developed based on the inverse velocity Method.

\subsection{SLO}

For both NTD and CEPN, interpreting the SLO method was very sensitive to data averaging for velocity calculation and also the choice of data for use in the slope calculation, as such the predicted date can vary significantly from day to day. In addition, many of the predicted dates were either beyond the actual date or in the past.

The SLO method clearly identified several trends that are easy to define for both the case studies. As such it may be useful in determining changes in trend even though the interpretation of data is more complicated.

The abstract nature of the SLO graphs and the need for data averaging to calculate velocity would render it difficult to use the SLO method for evacuation purposes.

\section{Conclusion}

None of the methods tested were able to provide a useful prediction of the failure date in the long term. In the case of CUSUM, this is not surprising as the authors of CUSUM, Sarunic and Lilly (2009), make no claim about CUSUMS' ability to predict failure date at any scale. Short term predictions, projecting forward once the final destabilising trend has been established showed some promise but require better understanding for decision making. All three methods allowed trends to be determined although the CUSUM and SLO methods are arguably more sensitive to trend changes, hence more useful. Due to the need for data averaging none of the methods are suitable as evacuation triggers as the response will be delayed.

\section{Acknowledgement}

We thank Rio Tinto Iron Ore for allowing us to use and publish this information.

\section{References}

Fukada, N., Ishijima, Y. and Fujii, Y. (1999) A Study on Prediction of Failure Time of Shikotsu Welded Tuff and Shirahama Sandstone in Creep Tests, s.l., in Proceedings Spring Meeting of MMIJ Hokkaido Branch, Mining and Materials Processing Institute of Japan, Hokkaido, pp. 23-24.

Fukuzono, T. (1985) A new method for predicting the failure time of a slope, Japan Landslide Society, Tokyo, pp. 145-150.

Mufundirwa, A. and Fujii, Y. (2008) New methods for prediction of geomechanical failure-time, in Proceedings Korean Rock Mechanics Symposium 2008, 22 October 2008, Chonnam National University, Gwangju, Korea, Korean Rock Mechanics Society, pp. 183-190. 
Mufundirwa, A. and Fujii, Y. (2010) Prediction of rock mass failure-time of geo-hazards, in Proceedings Rock Mechanics in Civil and Environmental Engineering, J. Zhao, V. Labiouse, J.P. Dudt and J.F. Mathier (eds), 15-18 June 2010, Lausanne, Switzerland, Taylor \& Francis, London, pp. 567-570.

Rose, N.D. and Hungr, O. (2007) Forecasting potential rock slope failure in open pit mines using the inverse velocity method, International Journal of Rock Mechanics and Mining Sciences, Vol. 44, Elsevier, pp. 308-320.

Sarunic, W. and Lilly, P.A. (2006) The Use of CUSUMS as a Tool to aid in the Interpretation of Slope Monitoring Data with Specific Examples from an Operating Open Pit Mine, in Proceedings International Symposium on Stability of Rock Slopes in Open Pit Mining and Civil Engineering, 3-6 April 2006, Cape Town, South Africa, The South African Institute of Mining and Metallurgy, Cape Town, pp. 231-238. 